\title{
SISTEMAS DE EVALUACIÓN Y METODOLOGÍAS DOCENTES Y SU INCIDENCIA EN LAS COMPETENCIAS GENÉRICAS (EMICOG ESTUDIANTES)
}

\author{
Judit García-Martín \\ Universidad de Salamanca \\ jgarm@unileon.es \\ Jesús N. García \\ Universidad de León/Universidad de la Costa \\ Alicia J. Inciarte \\ Universidad de la Costa (Colombia) \\ Freddy V. Marín \\ Universidad de la Costa (Colombia) \\ Edgardo R. Sánchez \\ Universidad de la Costa (Colombia) \\ Marcial E. Conde \\ Universidad de la Costa (Colombia)
}

Fecha de Recepción: 11 Enero 2019

Fecha de Admisión: 30 Abril 2019

\section{RESUMEN}

Las distintas reformas estructurales, organizativas y metodológicas que ha sufrido el Espacio de Educación Superior, han provocado cambios sustanciales en el modelo educativo imperante en el ámbito universitario y por consiguiente, en los planes de estudios. Bajo este prisma, en la presente comunicación se describe el diseño de un cuestionario ad hoc online, EMICOG - versión estudiantes, mediante la herramienta web, SurveyMonkey. Con él, se pretenden determinar y analizar las percepciones y creencias de utilidad futura que los alumnos tienen acerca de distintas metodologías docentes aplicadas en diversas asignaturas de grados y másteres impartidos en la Universidad de la Costa (Colombia). Al mismo tiempo que se examinan las formas de valoración o evaluación de las actividades transversales efectuadas, prestando especial atención a las competencias genéricas tales como la lectura crítica y la construcción textual. En este sentido, el EMICOG - versión estudiantes cuenta con ocho categorías claramente diferenciadas: i) datos de carácter general, ii) técnicas y estrategias instruccionales, iii) recursos utilizados en la evaluación y enseñanza de la asignatura, iv) variables psicoeducativas, v) necesidades formativas, vi) procesos de estudio y de aprendizaje, vii) actitudes hacia el aprendizaje y la evaluación y, viii) ansiedad hacia el aprendizaje y la evaluación, que son examinadas mediante escalas tipo Likert de 5 o 7 puntos. Por ello, la aplicación del mismo a 2275 estudiantes de pregrado de la Universidad de la Costa permitirá extraer perfiles dife- 


\section{SISTEMAS DE EVALUACIÓN Y METODOLOGÍAS DOCENTES Y SU INCIDENCIA EN LAS COMPETENCIAS GENÉRICAS (EMICOG ESTUDIANTES)}

renciales y diseñar estrategias de actuación instruccional exitosa para la mejora de los resultados académicos en las pruebas nacionales de Saber Pro, a las que se presentarán los discentes colombianos al finalizar sus estudios universitarios. Se trata de un Proyecto de Investigación Competitivo CONV-ÍNDEX Núm. 13-2018, Código Proyecto INV. 150-01-007-13 Universidad de la Costa (Colombia) - Universidad de León (España). Evaluado por la ACAC (MEN Colombia).

Palabras clave: competencias; instrumento; estudiantes; variables psicológicas

\section{ABSTRACT}

\section{Assessment systems and teaching methodologies and their incidence in generic compe- tences (emicog - students).}

The various structural, organizational and methodological reforms that the Higher Education Area has undergone have caused substantial changes in the prevailing educational model in the university sphere and, consequently, in the study plans. With this in mind, the present communication describes the design of an online ad hoc questionnaire, EMICOG - students version, through the web tool, SurveyMonkey. Therewith, it is intended to determine and analyze the perceptions and beliefs of future usefulness that students have about different teaching methodologies applied in various subjects studied of degrees and master's degrees taught at the Universidad de la Costa (Colombia). At the same time, the forms of assessment or evaluation of the transversal activities carried out are examined, paying special attention to generic competences such as critical reading and textual construction. In this sense, the EMICOG - students version, has eight clearly differentiated categories: i) general data, ii) instructional strategies and techniques, iii) resources used in the evaluation and teaching of the subject, iv) psychoeducational variables, v) training needs, vi) study and learning processes, vii) attitudes towards learning and evaluation and, viii) anxiety towards learning and evaluation, which are examined using 5 or 7 point Likert scales. Therefore, the application of the same to 2275 undergraduate students of the Universidad de la Costa will allow extracting differential profiles and designing strategies of successful instructional action for the improvement of the academic results in the national tests of Saber Pro, to which the Colombian students will present themselves at the end his university studies. Competitive Research Project CONV-ÍNDEX No. 13-2018, Project Code INV. 150-01-007-13 Universidad de la Costa (Colombia) - University of León (Spain). Evaluated by the ACAC (MEN Colombia).

Keywords: skills; instrument; students; psychological variables

\section{INTRODUCCIÓN}

En la educación superior actual se aboga por la adquisición de una amplia variedad de competencias (Barnett, 2001; Díaz-Florez, 2018) que promueven en los estudiantes el metaaprendizaje (Rocosa, Sangrá y Cabrera, 2018; Steffens, 2015), el lifelong learning (Horrigan, 2016; Quendler y Lamb, 2016; Steffens, 2015) así como el academic engagement o sentimiento de compromiso / implicación por el aprendizaje (Merino, Hontangas y Petrides, 2018) como alternativa a la mera asimilación de conocimientos teóricos. Esto unido a las distintas reformas estructurales, organizativas y metodológicas que ha sufrido el espacio de educación superior (Arias et. al., 2018), han provocado cambios sustanciales en el modelo educativo imperante en el ámbito universitario (García-Martín y García-Sánchez, 2017; García-Martín y García-Sánchez, 2018) y por consiguiente, en los planes de estudios (García, Marbán, de Caso, Pacheco, Robledo, Álvarez, García-Martín y García-Martín, 2011).

En esta línea, y atendiendo a lo observado en investigaciones previas, la motivación (García, de Caso, Arias y Fidalgo, 2005), el sistema de atribuciones (Balduzzi, 2010), las estrategias de afrontamiento (Morales, 2018), Ios estilos de aprendizaje (Gutiérrez, 2018; Gutiérrez, García, Vivas, Santizo, 
Alonso y Arranz, 2011; Robledo, García, Díez, Álvarez, Marbán, de Caso, Fidalgo, Arias, y Pacheco, 2010), la inteligencia emocional (Merino, Hontangas y Petrides, 2018), la autorregulación (Cheng y Lin, 2018; Doménech-Betoret, Abellán-Roselló y Gómez-Artiga, 2017; Fidalgo y García, 2008; Li, Ye, Tang, Zhou y Hu, 2018), las habilidades emocionales de los discentes (Gutiérrez, 2018; Morales, 2018) y los social media (García-Martín y García-Sánchez, 2013; García-Martín y García-Sánchez, 2015)), se han convertido en los promotores de la calidad educativa, influyendo positiva o negativamente en el rendimiento académico de los escolares (Doménech-Betoret et al., 2017; GarcíaMartín y Cantón, 2018; Karagiannopoulou, Milienos y Athanasopoulos, 2018).

Por todo ello, en el presente artículo se describe el diseño de un cuestionario ad hoc online, EMICOG - versión estudiantes, mediante la herramienta web, SurveyMonkey. Con su aplicación, se pretenden determinar y analizar las percepciones y creencias de utilidad futura que los alumnos tienen acerca de distintas metodologías docentes aplicadas en diversas asignaturas cursadas en titulaciones de la Universidad de la Costa (Colombia). Al mismo tiempo que se examinan las formas de valoración 0 evaluación de las actividades transversales efectuadas, prestando especial atención a las competencias genéricas comunicativas de lectura crítica y construcción textual.

\section{METODOLOGÍA}

\section{Participantes}

El pasado mes de Marzo de 2019, se envió el cuestionario ad hoc online, EMICOG - versión estudiantes, a los discentes de pregrado de la Universidad de la Costa (Colombia), de los cuales 3647, con edades comprendidas entre los 16 y 62 años, decidieron participar voluntariamente. Si bien, únicamente 2775 ( $\mathrm{N}=1336$ hombres y 1439 mujeres) completaron todos los ítems (véase tabla 1).

Tabla 1

Distribución de los participantes por género y facultad

\begin{tabular}{|c|c|c|c|c|c|c|}
\hline $\begin{array}{l}\text { Género/ } \\
\text { Facultad }\end{array}$ & $\underset{\text { ra }}{\text { Arquitectu }}$ & $\begin{array}{c}\text { Ciencias } \\
\text { Económica } \\
\text { s } \\
\end{array}$ & $\begin{array}{c}\text { Ciencias } \\
\text { Humanas } \\
\text { y Sociales } \\
\end{array}$ & $\begin{array}{c}\text { Derec } \\
\text { ho }\end{array}$ & $\begin{array}{c}\text { Ingenierí } \\
\mathbf{a}\end{array}$ & $\begin{array}{c}\text { Total } \\
\text { del } \\
\text { Género } \\
\end{array}$ \\
\hline Hombres & 78 & 207 & 55 & 55 & 933 & 1336 \\
\hline $\begin{array}{c}\text { Edad } H \\
\text { M } \\
\text { (DT) }\end{array}$ & $\begin{array}{c}21 \text { años } \\
(3.11)\end{array}$ & $\begin{array}{c}23 \text { años } \\
(5.11)\end{array}$ & $\begin{array}{c}22 \text { años } \\
(3.82)\end{array}$ & $\begin{array}{c}25 \\
\text { años } \\
(7.18)\end{array}$ & $\begin{array}{c}22 \text { años } \\
(4.51)\end{array}$ & $\begin{array}{c}22 \text { años } \\
(4.70)\end{array}$ \\
\hline Mujeres & 58 & 395 & 299 & 78 & 597 & 1439 \\
\hline $\begin{array}{c}\text { Edad } M \\
\text { M } \\
\text { (DT) }\end{array}$ & $\begin{array}{c}21 \text { años } \\
(3.73)\end{array}$ & $\begin{array}{c}23 \text { años } \\
(5.49)\end{array}$ & $\begin{array}{c}22 \text { años } \\
(4.11)\end{array}$ & $\begin{array}{c}23 \\
\text { años } \\
(5.96)\end{array}$ & $\begin{array}{c}21 \text { años } \\
(3.74)\end{array}$ & $\begin{array}{c}22 \text { años } \\
(4.60)\end{array}$ \\
\hline $\begin{array}{l}\text { Total de la } \\
\text { Facultad }\end{array}$ & 136 & 602 & 354 & 133 & 1530 & 2775 \\
\hline $\begin{array}{c}\text { Edad Total } \\
\text { M } \\
\text { (DT) }\end{array}$ & $\begin{array}{c}21 \text { años } \\
(3.37)\end{array}$ & $\begin{array}{c}23 \text { años } \\
(5.36)\end{array}$ & $\begin{array}{c}22 \text { años } \\
(4.06)\end{array}$ & $\begin{array}{c}24 \\
\text { años } \\
(6.54)\end{array}$ & $\begin{array}{c}22 \text { años } \\
(4.26)\end{array}$ & $\begin{array}{c}22 \text { años } \\
(4.65)\end{array}$ \\
\hline Min.-máx. Edad & $16-33$ & $16-51$ & $16-43$ & $16-50$ & $16-62$ & \\
\hline
\end{tabular}


Además, el $13,5 \%$ de los estudiantes participantes [ $\mathrm{N}=375$ (210 hombres y 165 mujeres)] indican trabajar a tiempo parcial, el 20,4\% [N = 566 (287 hombres y 279 mujeres)] a tiempo completo y el $66,1 \%$ no trabajaba [ $\mathrm{N}=1834$ (839 hombres y 995 mujeres)].

Igualmente, en relación a la estratificación socioeconómica, el 26,4\% de los encuestados [N = 732 (339 hombres y 393 mujeres)] manifiestan pertenecer al primer estrato denominado bajo-bajo; el $36 \%$ [N = 999 (480 hombres y 519 mujeres)] al segundo o bajo, 28,3\% [N = 785 (381 hombres y 401 mujeres)] al tercero o medio-bajo, el 7,4\% [N = 205 (108 hombres y 97 mujeres)] al cuarto 0 medio, el $1,4 \%$ [N = 40 (19 hombres y 21 mujeres)] al quinto 0 alto y el $0,5 \%$ [N = 14 (6 hombres y 8 mujeres)] restante al sexto 0 alto-alto (véase figura 1).

Figura 1. Descripción de la muestra por estratos económicos

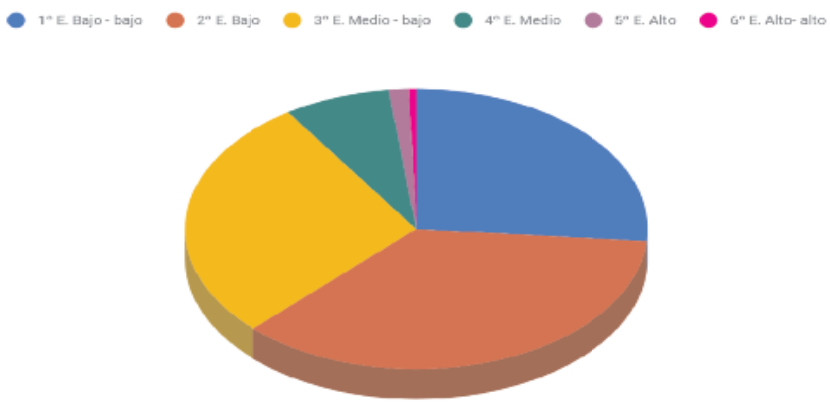

En cuanto a la distribución por semestres en base a la facultad, por un lado, la mayor parte de los estudiantes encuestados de ingeniería están matriculados en asignaturas de los últimos cinco semestres y por otro, el porcentaje más elevado de los de ciencias económicas, humanas y sociales es en el quinto. Sin embargo, los encuestados de derecho comprenden todos los semestres con una medida más o menos homogénea (véase figura 2).

Figura 2. Descripción de la muestra por semestres y facultades

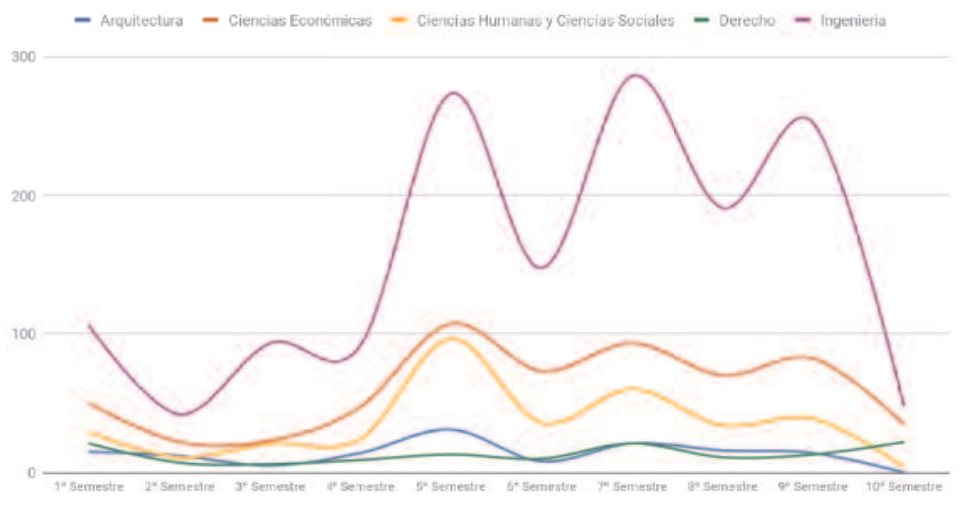




\section{Evaluación y metodologías docentes y su incidencia en las competencias genéricas, EMICOG - versión estudiantes}

A continuación, se describe el cuestionario ad hoc en línea, EMICOG - versión estudiantes, con el que se examinan las competencias desarrolladas y trabajadas en diferentes asignaturas de diversos grados y másteres de la Universidad de la Costa (Colombia). Este ha sido diseñado a través de la herramienta de la web 2.0, SurveyMonkey por varios motivos tales como la versatilidad, la usabilidad, la lógica de exclusión de preguntas, los saltos de página, la distribución aleatoria de bloques, la exportación de los datos en distintos formatos y las funciones avanzadas de análisis.

\section{Variables analizadas en el EMICOG - versión estudiantes}

EI EMICOG - versión estudiantes, recoge quince aspectos psicoeducativos: i) la presentación, ii) los datos de carácter general, iii) las técnicas y estrategias instruccionales, iv) la evaluación, v) la enseñanza, vi) la autoeficacia, vii) la inteligencia emocional, viii) la motivación, ix) las atribuciones, $\mathrm{x}$ ) las habilidades sociales, xi) las estrategias de afrontamiento, xii) las necesidades formativas, xiii) los estilos de pensamiento, xiv) las actitudes, preferencias, gustos y xv) la ansiedad. Si bien, estos aparecen agrupados en ocho categorías claramente diferenciadas: a) datos de carácter general, b) técnicas y estrategias instruccionales, c) recursos utilizados en la evaluación y enseñanza de la asignatura, d) variables psicoeducativas, e) necesidades formativas, f) procesos de estudio y de aprendizaje, g) actitudes hacia el aprendizaje y la evaluación y, h) ansiedad hacia el aprendizaje y la evaluación, que se detallan en la siguiente sección (véase tabla 2).

\section{Datos de carácter general}

En esta sección se incluye la pertinente presentación del estudio, en la que se expone el objetivo de la investigación, la duración estimada y se solicita la valiosa colaboración de los estudiantes, haciendo en todo momento, especial mención, a que ésta es totalmente voluntaria. En este sentido, y atendiendo al código deontológico de cualquier investigación científica, se hace plausible que únicamente se tendrán en cuenta los datos para análisis estadísticos globales con el fin de inferir patrones 0 tendencias sin interés particular o personal. Tras ello, se pregunta a cada uno de los encuestados si desean participar o no en el estudio (consentimiento informado). De modo que a aquellos que seleccionan la opción "Sí", se les requiere algunos datos demográficos tales como: nombre, apellidos, número de identificación, fecha de nacimiento, edad, género, estrato social familiar, carrera actual, semestre, facultad, departamento, si está compatibilizando los estudios con alguna actividad laboral, y en caso afirmativo, en qué rango. Igualmente, se les solicita indicar el nombre de la asignatura del semestre en la que han realizado, en base a su juicio, un número mayor de actividades de evaluación relacionadas con el uso de la construcción textual y/o lectura crítica, las asignaturas en las que su interés ha sido mayor así como el promedio de calificaciones del semestre...

Tras ello, se les pregunta mediante una escala tipo Likert de 5 puntos (ninguno, poco, ni poco ni mucho, bastante y mucho) sobre el conocimiento e interés que tienen en torno a competencias genéricas como la lectura crítica y la construcción textual.

\section{Técnicas y estrategias instruccionales}

Sobre esta categoría, se pide a los participantes que indiquen en base a una escala tipo Likert de 5 puntos (nunca, casi nunca, a veces, casi siempre y siempre) si alguna vez han requerido realizar en sus asignaturas, tareas como la elaboración de resúmenes y/o esquemas, la toma de apuntes, la construcción de mapas conceptuales, la realización de anotaciones, de auto-preguntas, acrósticos... para completar la información, para recordarla y/o para guiar los procesos de construcción textual tanto en el momento de planificación como de revisión. 
De igual modo, se les solicita que indiquen en base a la misma escala, si han utilizado en la asignatura objeto de análisis, diferentes tipologías textuales tales como los textos argumentativos, los de comparación y contraste, los de causa-efecto y/o problema-solución, los ensayos, las reseñas bibliográficas, las revisiones sobre el estado de la cuestión, tanto a la hora de estudiar un tema como a la de evaluar el aprendizaje. Además de concretar las percepciones que estos tienen acerca de la utilidad de dichas tipologías en la asignatura en base a otra escala tipo Likert de 5 puntos (muy baja, baja, ni baja ni alta, alta y muy alta).

Asimismo, se les insta en torno a los procesos psicológicos superiores implicados en cualquier tarea de composición escrita con independencia de la tipología textual: planificación, edición y revisión. Por ejemplo, se les pregunta si a la hora de planificar sus textos tienen en cuenta a los destinatarios del mismo, es decir, a la audiencia; si usan técnicas de escritura tales como guías o formularios y si revisan la calidad global y el orden de las ideas.

También, se les solicita que indiquen si en las asignaturas objeto de análisis utilizan preferiblemente como medio para implementar las estrategias de aprendizaje, la escritura a mano, los procesadores de textos, las herramientas de internet (blogs, wikis...) tanto a la hora de estudiar, de trabajar como de aprender, atendiendo a una escala tipo Likert de 5 puntos (nunca, casi nunca, a veces, casi siempre y siempre).

\section{Recursos utilizados en la evaluación y enseñanza de la asignatura}

En esta categoría, se les pide que señalen, si alguna vez, en la asignatura seleccionada, se han implementado procesos de enseñanza que incluyan estrategias y técnicas orientadas a: i) la extracción de ideas principales y de conclusiones e inferencias no explícitas, ii) la vinculación de ideas con conocimientos previos, iii) la aplicación de soluciones y otros aspectos, iv) la explicación del contenido básico de alguna lectura y v) la recuperación de la información leída previamente sin volver a consultar. Igualmente, se les pregunta si alguna vez, en la asignatura objeto de análisis se han implementado tareas como la lectura de algún texto argumentativo, de comparación y contraste, de causa-efecto, de ensayos, de alguna reseña bibliográfica... Al mismo tiempo que se les pregunta acerca del medio en el que se encontraban dichos documentos: herramientas web (blogs, wikis...), bases de datos digitales, en audiolibros o videodocumentos, en formato tradicional...

Antes de concluir dicha categoría, se les insta a determinar en qué medida, siguiendo la misma escala tipo Likert de 5 puntos, el docente de la asignatura utiliza diversos recursos para evaluar. Entre estos, se valoran los exámenes escritos de preguntas cortas, los de ensayo, las pruebas objetivas escritas, los análisis de trabajos escritos, la participación en debates, los análisis de presentaciones, los escritos de aplicaciones prácticas, los análisis escritos de lecturas de casos prácticos, de publicaciones científicas, la autoevaluación del aprendizaje, la coevaluación..., la elaboración y contribución en wikis y blogs que abordan algún problema nuclear de la asignatura... Esta escala se completa con una pregunta abierta en la que se les solicita que expliquen brevemente el sistema de evaluación de la asignatura.

Y para terminar, se les pide que determinen en qué medida se usan diferentes recursos en el proceso de enseñanza de los contenidos, tales como las lecciones magistrales, las charlas y conferencias, las lecturas y elaboración de textos argumentativos, las actividades de trabajo de campo, los casos prácticos, los proyectos, los debates, las dinámicas grupales, la solución de problemas, las clases con ordenador y herramientas virtuales en la nube... Igualmente, esta escala se completa con una pregunta abierta en la que se les solicita que describan brevemente la metodología docente empleada en la asignatura. 


\section{Variables psicoeducativas}

En esta sección, se valoran y analizan diferentes variables psicoeducativas mediante escalas tipo Likert de 7 puntos. Se examina la autoeficacia mediante quince expresiones del tipo: “¿En qué medida se siente capaz, en la asignatura, de resolver tareas y actividades de construcción textual?", la inteligencia emocional mediante siete ítems similares a: "Cuando alguien le cuenta algo que le ha sucedido, ¿lo siente como si le hubiera pasado a usted?", la motivación con tres preguntas del tipo: “¿Hace las cosas por la satisfacción que le reporta conseguirlas?”, las atribuciones con dos ítems, las habilidades sociales mediante ocho afirmaciones en las que se tienen que indicar el grado de acuerdo o desacuerdo y las estrategias de afrontamiento, mediante trece preguntas del tipo: "Cuando usted se encuentra en alguna dificultad, ¿utiliza el sentido del humor para restarle importancia?"

\section{Necesidades formativas}

En esta categoría, los estudiantes encuestados expresan tanto su grado de interés como de necesidad en recibir formación sobre la construcción de textos académicos y lectura crítica para preparar algún aspecto de la asignatura objeto de análisis, el TFG y/o el TFM. De igual modo, sobre herramientas web tales como recursos disponibles en la universidad, Massive Open Online Course (M00Cs), Google Drive, bases de datos y aplicaciones móviles para: tomar apuntes, estar informado, citar fuentes bibliográficas, organizar el tiempo, eliminar distracciones... Todo ello, siguiendo una escala tipo Likert de 5 puntos (nada necesario, poco necesario, ni poco ni mucho, necesario y muy necesario).

\section{Procesos de estudio y de aprendizaje}

En esta sección, se incluye el Cuestionario de Evaluación de los Procesos de Estudio y Aprendizaje (CEPEA) desarrollado por Barca Lozano en 1999 con el que delimitar los aspectos de tipo motivacional y estratégico que los estudiantes participantes desarrollan cuando realizan sus tareas de aprendizaje. En esta línea, se presentan 42 afirmaciones sobre las que los estudiantes encuestados tienen que indicar su grado de acuerdo o desacuerdo en base a una escala tipo Likert de 5 puntos; proporcionando de este modo, información sobre seis subescalas en el factor l: tres de motivación (superficial, profunda y de logro), tres de estrategias de aprendizaje (superficial, profundo y de logro) y en el factor II, otras tres escalas de enfoques de aprendizaje (superficial, profundo y de logro) que integran a los motivos y estrategias y por último los dos compuestos de enfoques (superficial-logro y profundo-logro).

\section{Actitudes hacia el aprendizaje y la evaluación}

En esta categoría, se les solicita responder a un total de veinte ítems (diez sobre cada competencia transversal) de menos a más sobre la coincidencia con sus gustos 0 hábitos. En este sentido, por ejemplo, en cuanto a la construcción textual se les plantea indicar su grado de acuerdo con: "Me gusta escribir textos (ensayos, trabajos..." y en relación a la lectura crítica con: "Leer textos es una pérdida de tiempo". Todo ello, en base a una escala tipo Likert de 5 puntos (nada, poco, ni poco ni mucho, algo y mucho).

\section{Ansiedad hacia el aprendizaje y la evaluación}

La última parte del cuestionario incluye 23 afirmaciones que versan sobre el grado de ansiedad que experimentan los discentes participantes, en concreto ocho sobre la evaluación como por ejemplo: "Me pongo nervioso/a cuando pienso en un examen escrito el día anterior", tres sobre la temporalidad como: "Me siento nervios/a cuando pienso en el examen escrito que tengo la semana pró- 
xima", cuatro en torno a la comprensión de tareas de construcción textual como: "Me pongo nervioso/a cuando pienso en comprender la redacción de un ensayo"y dos de lectura crítica como: "Me pone nervioso/a intentar comprender una lectura". A estos se añade, otros dos sobre documentos oficiales: "Me siento nervioso/a cuando tengo que hacer escritos oficiales: reclamaciones, formularios..." y otros cuatro generales del tipo: "Estudiar textos o construir textos son de las tareas que más temo".

Tabla 2

Síntesis de las categorías del cuestionario ad hoc, EMICOG - versión estudiantes

\begin{tabular}{|c|c|c|c|c|c|c|c|c|c|c|}
\hline $\begin{array}{l}\text { Instrumento / } \\
\text { Constructo }\end{array}$ & Subprueba & Ítems & $\alpha$ & $\begin{array}{l}\text { Núm. } \\
\text { Factores }\end{array}$ & Determinante & \begin{tabular}{|c|} 
Kaiser- \\
Meyer- \\
Olkin \\
Adecuación \\
muestreo
\end{tabular} & $\begin{array}{c}\text { Prueba } \\
\text { esfericidad } \\
\text { Bartlett }\end{array}$ & $\begin{array}{c}C R / \\
\omega\end{array}$ & $A V E$ & $\begin{array}{c}\text { Estudios previos de } \\
\text { validación e } \\
\text { implementación }\end{array}$ \\
\hline \multicolumn{11}{|c|}{ DG - Datos generales ( $N^{o}$ items 25$)$} \\
\hline $\begin{array}{c}\text { Datos } \\
\text { demográficos }\end{array}$ & DG_DD & 13 & & & & & & & & \multirow{2}{*}{$\begin{array}{l}\text { (Arias-Gómez et al., } \\
\text { 2018; Barnet, 2001; } \\
\text { CUC, 2018; Díaz- } \\
\text { Flórez, 2018; García- } \\
\text { Martín y García- } \\
\text { Sánchez, 2017; } \\
\text { OECD, 2016) }\end{array}$} \\
\hline Interés & DG-INT & 4 & 0.899 & 3 & 0.001 & 0.891 & 0.001 & 0.926 & 0.756 & \\
\hline \multicolumn{11}{|c|}{ TE - Técnicas y estrategias instruccionales ( $N^{\circ}$ items 41$)$} \\
\hline \multirow[b]{3}{*}{$\begin{array}{l}\text { Construcción } \\
\text { textual }\end{array}$} & $\begin{array}{l}\text { Estrategias y } \\
\text { técnicas } \\
\text { instruccionales }\end{array}$ & 6 & \multirow[b]{3}{*}{0.978} & \multirow[b]{3}{*}{4} & \multirow[b]{3}{*}{0.001} & \multirow[b]{3}{*}{0.967} & \multirow[b]{3}{*}{0.001} & 0.879 & 0.447 & \multirow{3}{*}{$\begin{array}{c}\text { (Calderón, Parra y } \\
\text { Piñeros, 2018; De la } \\
\text { Paz, Monte-Sano, } \\
\text { Felton, Croninger, } \\
\text { Jackson y Worland } \\
\text { Piantedosi, 2016; } \\
\text { Graham, 2018; } \\
\text { Graham et al., 2018; } \\
\text { Klein y Boscolo, } \\
\text { 2015; Koster, } \\
\text { Tribushinina, Jong y } \\
\text { van den Bergh 2015; } \\
\text { Miller, Scott y } \\
\text { Mitigue, 2018; Li, } \\
\text { Ye, Tang, Zhou y }\end{array}$} \\
\hline & Procesos & 5 & & & & & & 0.92 & 0.48 & \\
\hline & Medio & 4 & & & & & & 0.926 & 0.678 & \\
\hline & & & & & & & & & & $\begin{array}{c}\text { Hu, 2018; OECD, } \\
\text { 2016; Robledo y } \\
\text { García, 2018; } \\
\text { Semeraro, Coppola, } \\
\text { Cassibba, y } \\
\text { Lucangeli, 2019; van } \\
\text { Ockenburg, van } \\
\text { Weijen, Rijlaarsdam, } \\
\text { 2019) }\end{array}$ \\
\hline \multirow{2}{*}{ Lectura crítica } & $\begin{array}{l}\text { Géneros } \\
\text { textuales }\end{array}$ & 7 & \multirow{2}{*}{0.929} & \multirow[b]{2}{*}{3} & \multirow{2}{*}{0.001} & \multirow{2}{*}{0.971} & \multirow[b]{2}{*}{0.001} & 0.95 & 0.476 & \multirow{2}{*}{$\begin{array}{c}\text { (Calderón, Parra y } \\
\text { Piñeros, 2018; } \\
\text { Connor et al., 2017; } \\
\text { Ciullo et al., 2016; } \\
\text { Graham et al., 2018; } \\
\text { Guiñazú, Aldo y } \\
\text { Zibelman, 2014; Li et } \\
\text { al., 2018; OECD, } \\
\text { 2016; Peng et al., } \\
\text { 2019; }\end{array}$} \\
\hline & Medio & 6 & & & & & & 0.922 & 0.501 & \\
\hline \multicolumn{11}{|c|}{ EVEN - Evaluación y enseñanza de la asignatura ( $N^{\circ}$ items 43$)$} \\
\hline $\begin{array}{l}\text { Evaluación de } \\
\text { la asignatura }\end{array}$ & EV & 22 & 0.945 & 3 & 0.001 & 0.971 & 0.001 & 0.91 & 0.591 & $\begin{array}{l}\text { (CEEDAR, 2019a; } \\
\text { 2019b; Ciullo et al., } \\
\text { 2016; De la Paz et } \\
\text { al., 2016; García et } \\
\text { al., 2005; García et } \\
\text { al., 2011; Graham et } \\
\text { al., 2018; } \\
\text { Karagiannopoulou, } \\
\text { Milienos y } \\
\text { Athanasopoulos, } \\
\text { 2018; Li, Ye, Tang, }\end{array}$ \\
\hline
\end{tabular}




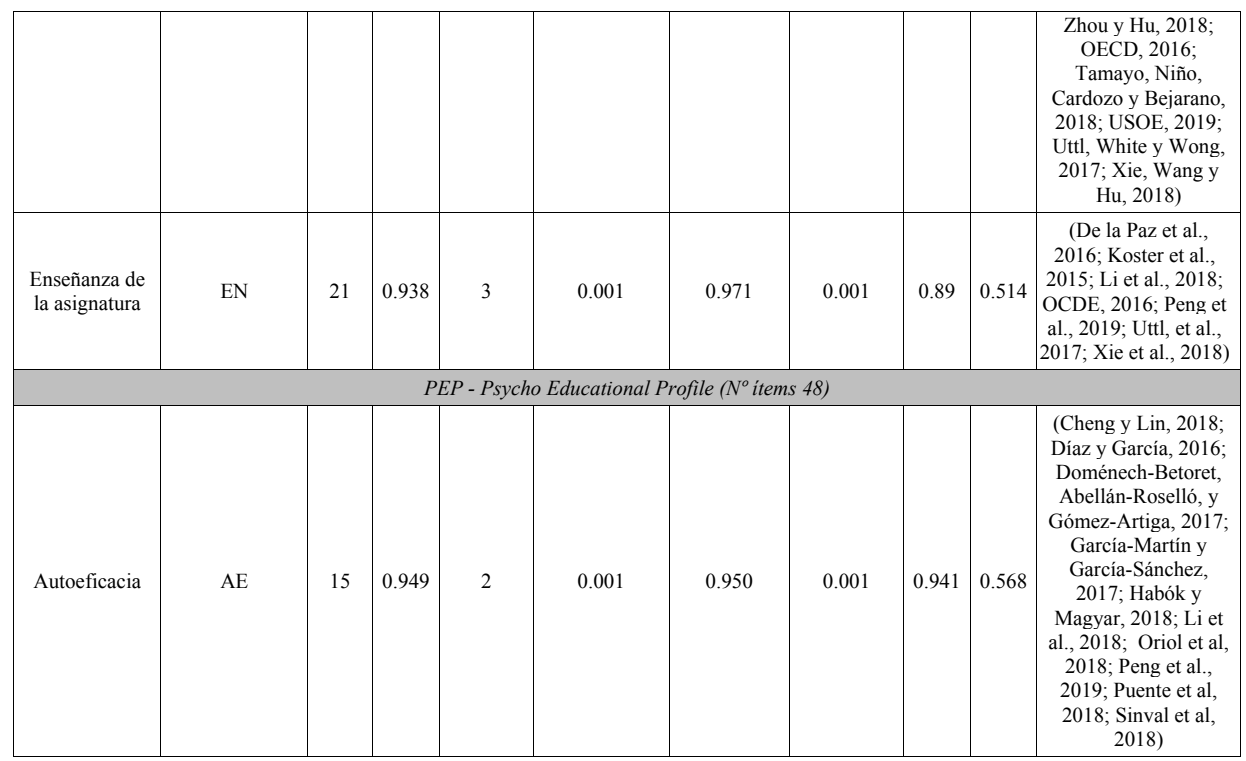

\begin{tabular}{|c|c|c|c|c|c|c|c|c|c|c|}
\hline $\begin{array}{l}\text { Dimensión } \\
\text { social }\end{array}$ & DS & 8 & 0.756 & & & & & 0.898 & 0.561 & $\begin{array}{c}\text { (Graham, 2018; } \\
\text { Habók y Magyar, } \\
\text { 2018; } \\
\text { Karagiannopoulou et } \\
\text { al., 2018 Klein y } \\
\text { Boscolo, 2015; Li et } \\
\text { al., 2018; Puente et } \\
\text { al, 2018; Romera et } \\
\text { al, 2018) }\end{array}$ \\
\hline $\begin{array}{c}\text { Inteligencia } \\
\text { emocional }\end{array}$ & PEP_IE & 7 & 0.807 & 3 & 0.099 & 0.824 & 0.001 & 0.873 & 0.5 & $\begin{array}{c}\text { (Doménech-Betoret } \\
\text { et al., 2017; Graham, } \\
\text { 2018; Habók y } \\
\text { Magyar, 2018; Klein } \\
\text { y Boscolo, 2015; Li } \\
\text { et al., 2018; Oriol et } \\
\text { al, 2018; Puente et al, } \\
\text { 2018; Romera et al, } \\
\text { 2018; Sinval et al, } \\
\text { 2018) }\end{array}$ \\
\hline Motivación & $\begin{array}{c}\text { MOT_ATR }+ \\
\text { TOT }\end{array}$ & 6 & 0.703 & & & & & 0.778 & 0.637 & $\begin{array}{l}\text { (Doménech-Betoret } \\
\text { et al., 2017; Graham, } \\
\text { 2018; Li et al., 2018) }\end{array}$ \\
\hline Atribuciones & & 2 & 0.464 & & & & & 0.775 & 0.607 & $\begin{array}{l}\text { (Doménech-Betoret } \\
\text { et al., 2017; Graham, } \\
\text { 2018; Li et al., 2018; } \\
\text { Puente et al, 2018) }\end{array}$ \\
\hline Afrontamiento & AFR & 13 & 0.628 & 3 & 0.007 & 0.862 & 0.001 & 0.875 & 0.64 & $\begin{array}{l}\text { (Graham, 2018; } \\
\text { Puente et al, 2018; } \\
\text { Sinval et al, 2018) }\end{array}$ \\
\hline
\end{tabular}




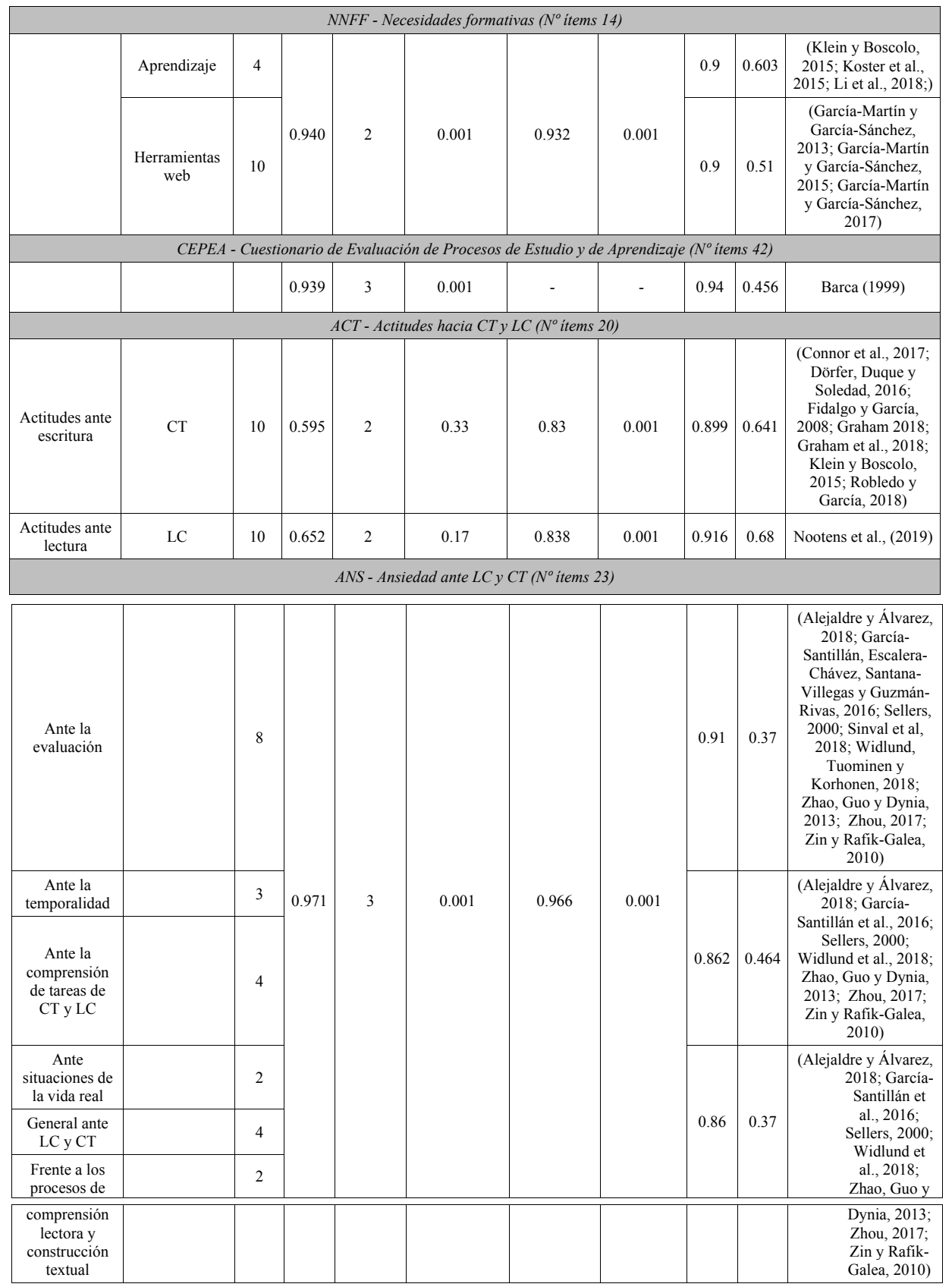




\section{DISCUSIÓN Y CONCLUSIONES}

El cuestionario, EMICOG- versión estudiantes, ha sido diseñado con el objetivo de determinar y analizar las percepciones y creencias de utilidad futura que los alumnos universitarios tienen acerca de las distintas metodologías docentes aplicadas en varias asignaturas cursadas.

Con los resultados obtenidos en la aplicación del mismo a la población de universitaria de pregrado de la Costa (Colombia), se tratará de extraer perfiles diferenciales y diseñar estrategias de actuación instruccional exitosa para la mejora de los resultados académicos en los Exámenes de Calidad de la Educación Superior (ECAES), que a partir de 2009 pasaron a denominarse pruebas a Saber Pro (Calderón, Parra y Piñeros, 2018), a las que se presentan los discentes colombianos que están en los últimos semestres de sus titulaciones. Éstas son realizadas por el Ministerio de Educación de Colombia (OECD, 2016) y tienen por objetivo conocer el nivel de rendimiento académico de las distintas instituciones educativas nacionales favoreciendo el establecimiento de rankings (CEEDAR, 2019a, 2019b).

En esta línea, estudios futuros versarán sobre la adaptación de este instrumento a otras poblaciones universitarias con la finalidad de establecer patrones diferenciales en base al género, la titulación, la edad, la asignatura cursada... para de este modo, poder ajustar los programas instruccionales a las demandas de la población universitaria, teniendo presentes en todo momento, las necesidades expresadas por éstos, así como sus características psicoeducativas (la autoeficacia, la motivación, la inteligencia emocional, las habilidades sociales...).

\section{REFERENCIAS BIBLIOGRÁFICAS}

Alejaldre, L., y Álvarez, E. (2018). Impacto de la ansiedad en la lectura en voz alta en el aula: estudio de estudiantes de ELE tailandeses de nivel A1. Tejuelo, 28, 219-252. doi: 10.17398/19888430.28.219

Arias, D. H., Díaz, O. C., Garzón, I., León, A. C., Rodríguez, S. P., y Valbuena, E. 0. (2018). Entre las exigencias de calidad y las condiciones de desigualdad: Formación inicial de profesores en Colombia. Bogotá: UPN.

Balduzzi, M. (2010). Procesos de atribución y autopercepción en estudiantes universitarios. Revista Intercontinental de Psicología y Educación, 12(1), 89-116.

Barca, A. (1999). Manual del Cuestionario de Evaluación de Procesos de Estudio y Aprendizaje para el alumnado universitario (escala CEPEA). A Coruña: Revista Galego-Portuguesa de Psicoloxia e Educación.

Barnet, R. (2001). Los límites de la competencia. El conocimiento, la educación superior y la sociedad. Barcelona: Gedisa.

Calderón, A., Parra, C. A., y Piñeros, M. A. (2018). Guía de orientación Saber Pro: Módulos de competencias genéricas. Bogotá, Colombia: Instituto Colombiano para la Evaluación de la Educación (Icfes).

CEEDAR. (2019a). Evidences Based Practices. CEEDAR innovation configurations. Tools for programs reforms. Recuperado de http://ceedar. education.ufl.edu/tools/innovation-configurations/

CEEDAR. (2019b). The CEEDAR Center Evidence Standards. Recuperado de http://ceedar.education.ufl.edu/wp-content/uploads/2014/08/Evidence-Based-Practices-guide.pdf

Cheng, Y., y Lin, Y. (2018). Validation of the short self-regulation questionnaire for Taiwanese College Students (TSSRQ). Frontiers in Psychology, 9,(259), 1-10. doi: 10.3389/fpsyg.2018.00259

Ciullo, S., Lembke, E. S., Carlisle, A., Thomas, C. N., Goodwin, M., y Judd, L. (2016). Implementation of evidence-based literacy practices in middle school response to intervention: an observation study. Learning Disability Quarterly, 39(1), 44-57. doi: 10.1177/0731948714566120 
Connor, C., Dombek, J., Crowe, C., Spencer, M., Tighe, E., Coffinger, S., Zagar, E., Wood, T., y Petscher, Y. (2017). Acquiring science and social studies knowledge in kindergarten fourth grade: conceptualization, design, implementation, and efficacy testing of content-area literacy instruction (CALI). Journal of Educational Psychology, 109(3), 301-320. doi: 10.1037/edu0000128

CUC. (2018). Autoinforme de Acreditación. Barranquilla: CUC

De la Paz, S., Monte-Sano, C., Felton, M., Croninger, R., Jackson, C., y Worland Piantedosi, K. (2016). A historical writing apprenticeship for adolescents: integrating disciplinary learning with cognitive strategies. Reading Research Quarterly, 52(1), 31-52. doi: 10.1002/rrq.147

Díaz-Flórez, 0. C. (2018). Las competencias en la educación superior. Debates contemporáneos. Tesis Doctoral. Bogotá: UPN

Doménech-Betoret, F., Abellán-Roselló, L., y Gómez-Artiga, A. (2017). Self-efficacy, satisfaction, and academic achievement: the mediator role of students' expectancy-value beliefs. Frontiers in Psychology, 8, 1-12. doi: 10.3389/fpsyg.2017.01193

Dörfer, C., Duque, U., y Soledad, G. (2016). Mediación de la actitud hacia las matemáticas en estudiantes de licenciatura en administración: un estudio piloto. VinculaTégica Efan, 2(1), 14821501.

Fidalgo, R., y García, J. N. (2008). Instrucción de la autorregulación y el autoconocimiento (metacognición) en la composición escrita. Barcelona: DaVinci Continental.

García, J. N., de Caso, A. M., Arias, O., y Fidalgo, R. (2005). La evaluación de prácticas universitarias y su aplicación en un enfoque innovador. Revista de educación, 337, 295-325.

García, J. N., Marbán, J., de Caso, A., Pacheco, D., Robledo, P., Álvarez, L., García-Martín, J., y García-Martín, E. (2011). Innovación en el EEES con metodologías activas. International Journal of Developmental and Educational Psychology, 3(1), 365-372.

García-Martín, J., y García-Sánchez (2013). Patterns of Web 2.0 tool use among young Spanish people Computers \& Education, 67, 105-120. doi: 10.1016/j.compedu.2013.03.03.003

García-Martín, J., y García-Sánchez, J. N. (2015). Use of Facebook, Tuenti, Twitter and Myspace among young Spanish people. Behaviour \& Information Technology, 34(7), 34-37. doi: 10.1080/0144929X.2014.993428

García-Martín, J., y García-Sánchez, J. N. (2017). Pre-service teachers' perceptions of the competence dimensions of digital literacy and of psychological and educational measures. Computers \& Education, 107, 54-67. doi: 10.1016/j.com pedu.2016.12.010

García-Martín, J., y García-Sánchez, J. N. (2018). The instructional effectiveness of two virtual approaches: Processes and product. Journal of Psychodidicatics, 23(2), 117-127. doi: 10.1016/j.psicoe.2018.02.003

García-Martín, S., y Cantón, I. (2018). Use of technologies and academic performance in adolescent students. Comunicar, 27(59), 73-81. doi: 10.3916/C59-2019-07

García-Santillán, A., Escalera-Chávez, M. E., Santana-Villegas, J. C., y Guzmán-Rivas, B. Y. (2016). Estudio empírico para determinar el nivel de ansiedad hacia la matemática en estudiantes universitarios. International Journal of Developmental and Educational Psychology, 1(2), 441-452.

Graham, S. (2018). A revised writer(s)-within-community model of writing. Educational Psychologist, 53(4), 258-279. doi: 10.1080/00461520.2018.1481406

Graham, S., Liu, X., Bartlett, B. Ng, C., Harris, K. R., Aitken, A., Barkel, A., Kavanauge, C., y Talukdar, J. (2018). Reading for writing: a meta-analysis of the impact of reading interventions on writing. Review of Educational Research, 88(2), 243-284. doi: 10.3102/0034654317746927

Graham, S., Liu, X., Ng, C., Bartlett, B., Harris, K. R., y Kavanauge, C. (2018). Effectiveness of liter- 
acy programs balancing reading and writing instruction: a meta-analysis. Reading Research Quarterly, 53(3), 279-304. doi: 10.1002/rrq.194

Guiñazú, M. R., Aldo, B., y Zibelman, V. (2014). La lectura en el ámbito universitario. Estrategias para intentar resolver un conflicto. Revista Facultad de Odontología, 7(1), 45-51.

Gutiérrez, M. (2018). Estilos de aprendizaje, estrategias para enseñar. Su relación con el desarrollo emocional y "aprender a aprender". Tendencias Pedagógicas, 31, 83-96.

Gutiérrez, M., García, J. L., Vivas, M., Santizo, J. A., Alonso, C., y Arranz, M. S. (2011). Estudio comparativo de los estilos de aprendizajes del alumnado que inicia sus estudios universitarios en diversas facultades de Venezuela, México y España. Learning Styles Review, 7(7), 35-62.

Habók, A., y Magyar, A. (2018). Validation of a self-regulated foreign language learning strategy questionnaire through multidimensional modelling. Frontiers in Psychology, 9, 1-11. doi: 10.3389/fpsyg.2018.01388

Horrigan, J. B. (2016). Lifelong learning and technology. Washington, District of Columbia: Pew Research Center.

Karagiannopoulou E., Milienos F. S., y Athanasopoulos, V. (2018). Associations between defense styles, approaches to learning, and achievement among university students. Frontiers in Psychology, 9, 1-10. doi: 10.3389/feduc.2018.00053

Klein, P. D., y Boscolo, P. (2015). Trends in research on writing as a learning activity. Journal of Writing Research, 7(3), 311-350. doi: 10.17239/ jowr-2016.07.03.01

Koster, M., Tribushinina, E., de Jong, P. F., y van den Bergh, H. (2015). Teaching children to write: a meta-analysis of writing intervention research. Journal of Writing Research, 7(2), 249-274. doi: 10.17239/jowr-2015.07.02.2

Li, J., Ye, H., Tang, Y., Zhou, Z., y Hu, X. (2018). What are the effects of self-regulation phases and strategies for Chinese students? A meta-analysis of two decades research of the association between self-regulation and academic performance. Frontiers in Psychology, 9, 1-13. doi: 10.3389/fpsyg.2018.02434

Merino, E., Hontangas, P., y Petrides, K. V. (2018). Career Adaptability Mediates the Effect of Trait Emotional Intelligence on Academic Engagement. Revista de psicodidáctica, 23(2), 77-85.

Miller, D. M., Scott, C. E., y McTigue, E. M. (2018). Writing in the secondary-level disciplines: A systematic review of context, cognition and content. Educational Psychology Review, 30(1), 83120. doi: 10.1007/s10648-016-9393-z

Morales, F. M. (2018). Estrategias de afrontamiento en una muestra de estudiantes universitarios. International Journal of Developmental and Educational Psychology, 2(1), 289-294.

Nootens, P., Morin, M. F., Alamargot, D., Gonçalves, C., Venet, M., y Labrecque, A. (2019). Differences in attitudes toward reading: a survey of pupils in Grade 5 to 8. Frontiers in Psychology, 9, 1-13. doi: 10.3389/fpsyg.2018.02773

OECD. (2016). La educación en Colombia. MEC: OECD. Recuperado de: https://www.mineducacion.gov.co/1759/articles-356787_recurso_1.pdf

Oriol, X., Miranda, R., Oyanedel, J. C., y Torres, J. (2017). The role of self-control and grit in domains of school success in students of primary and secondary school. Frontiers in Psychology, 8, 110. doi: $10.3389 /$ fpsyg.2017.01716

Peng, P., Wang, T., Wang, C., y Lin, X. (2019). A meta-analysis on the relation between fluid intelligence and reading/mathematics: effects of tasks, age, and social economics status. Psychological Bulletin, 145(2), 189-236. doi: 10.1037/bul0000182

Puente-Martínez, A., Páez, D., Ubillos-Landa, S., y Da Costa-Dutra, S. (2018). Examining the structure of negative affect regulation and its association with hedonic and psychological wellbeing. 
Frontiers in Psychology. 9, 1-10. doi: 10.3389/fpsyg.2018.01592

Quendler, E., y Lamb, M. (2016). Learning as a lifelong process - meeting the challenges of the changing employability landscape: competences, skills and knowledge for sustainable development. International Journal of Continuing Engineering Education and Lifelong Learning, 26(3), 1-15. doi: 10.1504/IJCE ELL.2016.078447

Robledo, P., García, J. N., Díez, C., Álvarez, L., Marbán, J., de Caso, A., Fidalgo, R., Arias, O., y Pacheco, D. (2010). Estilos de pensamiento y aprendizaje en estudiantes de magisterio y psicopedagogía: diferencias según curso y especialidad. Escritos de Psicología, 3(3), 27-36. doi: 10.5231/psy.writ.2010. 0707

Robledo, P., y García, J. N. (2018). Chapter 3: Description and Analysis of Strategy-Focused Instructional Models for Writing. En R. Fidalgo y T. Olive (Series Eds.) y R. Fidalgo, K. R. Harris, y M. Braaksma, (Vol Eds.), Studies in Writing Series: Vol. 34. Design Principles for Teaching Effective Writing (pp. 38-65). Leiden: Brill.

Rocosa, B., Sangrá, A., y Cabrera, N. (2018). La organización escolar y el desarrollo de la competencia de Aprender a Aprender: Un enfoque globalizador singular. REXE: Revista de estudios y experiencias en educación, 2(1), 31-51.

Romera, E. M., Herrera, M., Casas, J. A., Ortega, R., y del Rey, R. (2018). How much do adolescents cybergossip? Scale Development and Validation in Spain and Colombia. Frontiers in Psychology, 9, 1-11. doi: 10.3389/fpsyg.2018.00126

Sellers, V. (2000). Anxiety and reading comprehension in Spanish as a foreign language. Foreign Language Annals, 33(5), 512-521.

Semeraro, C., Coppola, G., Cassibba, R., y Lucangeli, D. (2019). Teaching of cursive writing in the first year of primary school: Effect on reading and writing skills. PIOS ONE, 14(2), 1-17. doi: 10.1371/journal.pone.0209978

Sinval, J., Pinto, A., Queirós, C., y Maroco, J. (2018). Work engagement among rescue workers: psychometric properties of the Portuguese UWES. Frontiers in Psychology, 8, 1-16. doi: 10.3389/fpsyg.2017.02229

Steffens, K. (2015). Competences, learning theories and M00Cs: recent developments in lifelong learning. European Journal of Education, 50(1), 41-59.

Tamayo, L. A., Niño, L. S., Cardozo, L. S., y Bejarano, 0. (2018). ¿Hacia dónde va la evaluación? Aportes conceptuales para pensar y transformar las prácticas de evaluación. Bogotá: IDEP

USOE. (2019). OSEP Ideas that Work. Office of Special Education Programs, EEUU: Department of Education.

Uttl, B., White, C. A., y Wong, D. (2017): Meta-analysis of faculty s teaching effectiveness: Student evaluation of teaching ratings and student learning are not related. Studies in Educational Evaluation, 54, 22-42. doi: 10.1016/j.stueduc.2016.08.007

Van Ockenburg, L., Daphne van Weijen, D., y Rijlaarsdam, G. (2019). Learning to write synthesis texts: a review of intervention studies. Journal of Writing Research, 10(3), 401-428. doi: 10.17239/jowr-2019.10.03.01

Widlund, A., Tuominen, H., y Korhonen, J. (2018). Academic well-being, mathematics performance, and educational aspirations in lower secondary education: changes within a school year. Frontiers in Psychology, 9, 1-20. doi: 10.3389/fpsyg.2018.00297

Xie C., Wang, M., y Hu, H. (2018). Effects of constructivist and transmission instructional models on mathematics achievement in mainland China: a meta-Analysis. Frontiers in Psychology, 9, 112. doi: $10.3389 /$ fpsyg.2018.01923

Zhao, A., Guo, Y., y Dynia, J. (2013). Foreign language reading anxiety: Chinese as a foreign lan- 
guage in the United States. The Modern Language Journal, 97(3), 764-778. doi: 10.1111/j.1540-4781.2013.12032.x

Zhou, J. (2017). Foreign language reading anxiety in a Chinese as a foreign language context. Reading in a Foreign Language, 9(1), 155-173.

Zin, M. Z., y Rafik-Galea, S. (2010). Anxiety and academic reading performance among Malay ESL learners. Journal of Pan-Pacific Association of Applied Linguistics, 14(2), 41-58. 
